\title{
Evaluation of Autonomic Nervous System Changes Using Short-term Heart Rate Variability During Apnea
}

\section{Baolin He}

China Medical University

\section{Wenyu Li}

Wuhan University Zhongnan Hospital

\section{Xiaotong Zhang}

China Medical University

\section{Yanan Wu}

China Medical University

\section{Jing Liu}

Wuhan University Zhongnan Hospital

\section{Lara M Brewer}

University of Utah

Lu Yu ( $\nabla$ lyu@cmu.edu.cn )

China Medical University https://orcid.org/0000-0002-2672-0116

\section{Research}

Keywords: Autonomic nervous system, Apnea, Short-term, Heart rate variability

Posted Date: May 21st, 2020

DOl: https://doi.org/10.21203/rs.3.rs-29549/v1

License: (c) (i) This work is licensed under a Creative Commons Attribution 4.0 International License. Read Full License 


\section{Title page}

2 Evaluation of Autonomic Nervous System Changes Using

3 Short-term Heart Rate Variability During Apnea

4 Baolin He, B.S. ${ }^{1 \#}$, Wenyu Li, M.S. ${ }^{2 \#}$, Xiaotong Zhang, B.S. ${ }^{1}$, Yanan Wu, B.S. ${ }^{1}$, Jing

$5 \quad$ Liu, M.S. ${ }^{2}$, Lara M. Brewer, Ph.D. ${ }^{3}$, Lu Yu, M.S. ${ }^{1}$

$6{ }^{1}$ Department of Biomedical Engineering, School of Fundamental Sciences, China

$7 \quad$ Medical University, Shenyang, Liaoning, P. R. China

$8{ }^{2}$ Department of Nuclear Medicine, Zhongnan Hospital of Wuhan University, Wuhan,

$9 \quad$ Hubei, China

${ }^{3}$ Department of Anesthesiology, University of Utah, Salt Lake City, Utah, USA

11 \# These authors contributed equally to the work

12 The correspondence should be addressed to Lu Yu; lyu@cmu.edu.cn

\section{Abstract}

14 Background: Frequent cessations of respiration can greatly increase the prevalence 15 rate of arrhythmia. It has been confirmed that cardiac activity is regulated by autonomic nervous system (ANS). And heart rate variability (HRV) is widely used as a method to evaluate the function of ANS. Therefore, we analyzed whether apnea can affect the balance and normal function of ANS using short-term HRV indices. 
Methods: Forty-five healthy subjects were asked to breathe normally and hold their breathing to simulate 10 times apnea. Thirty-six patients from the dataset of a sleep laboratory for the diagnosis of sleep disorders with 10 times apnea were included in analysis. We calculated short-term HRV indices of subjects in normal respiratory and apneic states, respectively.

Results: Compared with normal respiratory state, respiration cease would lead to the values of the mean-RR, nLF, LF/HF, and $\alpha 1$ were significantly increase whereas the values of rMSSD and nHF were significantly decrease.

Conclusions: Cessations of respiration would lead to an imbalance in function of ANS, as well as an increase in fractal characteristics of the heart. These changes in physiological state are likely to induce and cause the occurrence of arrhythmia, which is regulated by ANS.

Keywords Autonomic nervous system, Apnea, Short-term, Heart rate variability

\section{Background}

People who experience frequent apneic periods initiated by either voluntary or involuntary apnea are more prone to develop cardiac arrhythmia [1]. For example, voluntary breath-holding divers and underwater hockey players are more prone to cardiac arrhythmia [2]. More than $90 \%$ of patients with obstructive sleep apnea hypopnea syndrome, characterized by involuntary apnea during sleep, also have cardiac arrhythmia [3]. The incidence of frequent apneic periods may be a high risk factor for non-organic cardiac arrhythmia, which is a hallmark of a dysregulated autonomic nervous system (ANS) [4]. We studied whether apneic periods (both 
voluntary and involuntary apnea) can affect the balance and normal function of the ANS, exhibited by the presence of cardiac arrhythmia. The methods of heart rate variability (HRV), blood pressure monitoring, pulse transit time (PTT), catecholamine assay, and radionuclide imaging [5] can be adopted to evaluate the function of ANS. Compared with other methods, HRV has the advantages of being noninvasive, quantitative, and simple to calculate. It can analyze the dynamic changes of the ANS more accurately and better evaluate the cardiac sympatho-vagal balance. We hypothesized that HRV could be used to evaluate the effect of apnea on the function of the ANS.

\section{Results}

The results of study1 and study2 show that when apnea (either type) occurred, the values of mean-RR, nLF, LF/HF, and $\alpha 1$ increased and the values of rMSSD and nHF decreased. The HRV indices under different respiratory states in study1 were shown in Table 2 and Fig.3. Table 2 and Fig. 3 show that for the subjects in study1, comparing with normal respiratory state, under the state of voluntary apnea, the value of mean-RR (10 events of voluntary apnea) is much more significantly increased, and the values of $\mathrm{nLF}, \mathrm{LF} / \mathrm{HF}$, and $\alpha 1$ are significantly increased $(\geq 7$ events of voluntary apnea), whereas the value of rMSSD is significantly decreased (10 events of voluntary apnea), and the value of nHF is significantly decreased ( 8 events of voluntary apnea).

Table 2 Result of HRV during normal respiratory condition and voluntary apnea 
Normal

$\begin{array}{llllll}831.59 \pm 19.34 & 41.57 \pm 4.62 & 44.16 \pm 2.71 & 55.84 \pm 2.71 & 1.07 \pm 0.15 & 0.94 \pm 0.04\end{array}$

Respiratory State

$1.79 \pm 0.27 *$

$1.18 \pm 0.09 *$

$2^{\text {nd }}$ Voluntary Apnea

$873.36 \pm 21.95^{*}$

$20.62 \pm 1.83^{*}$

$52.43 \pm 2.79 *$

$47.57 \pm 2.79 *$

$1.53 \pm 0.21$

$1.18 \pm 0.07 *$

$3^{\text {rd }}$ Voluntary Apnea

$874.32 \pm 20.98 *$

$20.02 \pm 1.49 *$

$51.96 \pm 3.14 *$

$48.04+3.14 *$

$1.66 \pm 0.24 *$

$1.05 \pm 0.08$

$4^{\text {th }}$ Voluntary Apnea

$873.23 \pm 22.35 *$

$21.21 \pm 1.64 *$

$48.23 \pm 3.01$

$1.4 \pm 0.23$

$1.13 \pm 0.07 *$

$5^{\text {th }}$ Voluntary Apnea

$874.89 \pm 21.84 *$

$21.45 \pm 1.72 *$

$52.98 \pm 3.51 *$

$47.02 \pm 3.51 *$

$2.1 \pm 0.36^{*}$

$1.17 \pm 0.07 *$

$6^{\text {th }}$ Voluntary Apnea

$874.53 \pm 22.33 *$

$19.73 \pm 1.61 *$

$50.73 \pm 3.05^{*}$

$49.27 \pm 3.05 *$

$1.58 \pm 0.22 *$

$1.21 \pm 0.08 *$

$7^{\text {th }}$ Voluntary Apnea

$867.8 \pm 20.97 *$

$21.86 \pm 1.98 *$

$52.76 \pm 3.54 *$

$47.24 \pm 3.54 *$

$2.24 \pm 0.43 *$

$1.18 \pm 0.08 *$

$8^{\text {th }}$ Voluntary Apnea

$870.88 \pm 20.6^{*}$

$21.17 \pm 2.25^{*}$

$49.17 \pm 3.16$

$1.5 \pm 0.2$

$1.15 \pm 0.09 *$

$9^{\text {th }}$ Voluntary Apnea

$871.18 \pm 20.56^{*}$

$20.52 \pm 1.68 *$

$55.91 \pm 3.14 *$

$44.09 \pm 3.14^{*}$

$1.91 \pm 0.25 *$

$1.35 \pm 0.08 *$

$10^{\text {th }}$ Voluntary Apnea

$880.8 \pm 21.77 *$

$21.2 \pm 2.08 *$

$56 \pm 3.11^{*}$

$44 \pm 3.11 *$

$2 \pm 0.25 *$

$1.11 \pm 0.07$

(a)

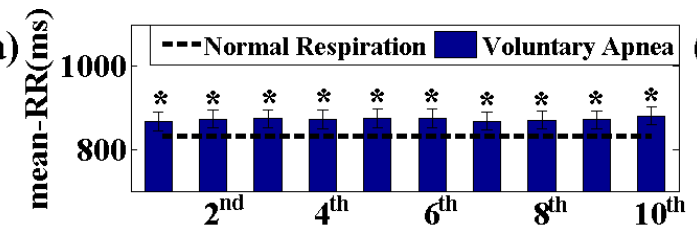

Voluntary Apneic Period(times)

(c)

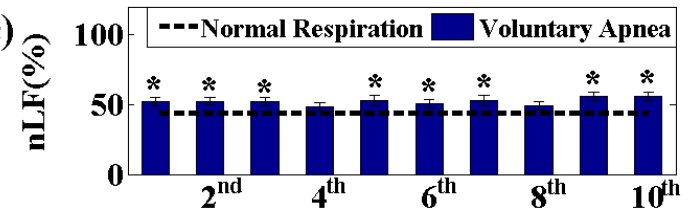

Voluntary Apneic Period(times)

(e) $\quad 6 \quad-1$-Normal Respiration $\quad$ Voluntary Apnea (f)

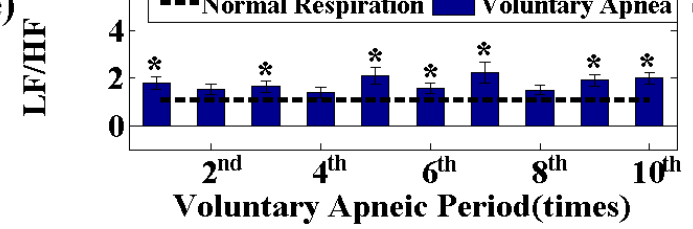

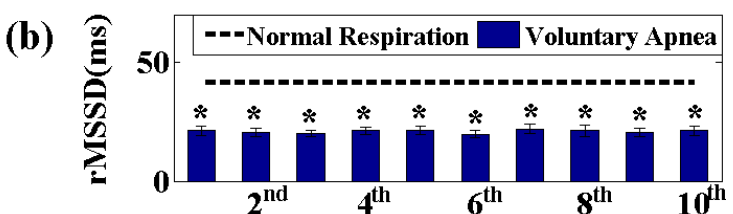
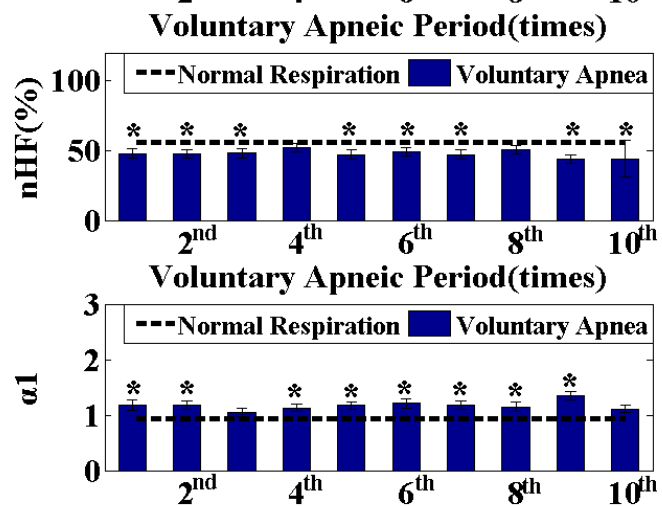

Voluntary Apneic Period(times) 
Fig. 3. The values of HRV during normal respiratory condition (dotted line) and voluntary apneic state (bar) in study1. (a) the value of Mean-RR, (b) the value of rMSSD, (c) the value of nLF, (d) the value of nHF, (e) the value of LF/HF, (f) the value of $\alpha 1$.

Values plotted are means \pm SE. Statistical significance between the voluntary apneas and normal breathing: $*(\mathrm{P}<0.05)$ significant.

The HRV indices in different respiratory states in study2 were shown in Table 3 and Fig.4. Table 3 and Fig.4 show that for the subjects of study2, comparing with normal respiratory state, under the apneic state, the values of LF/HF and $\alpha 1$ are significantly increased (10 events of apnea), and the values of mean-RR and nLF are significantly increased ( 8 events of apnea), whereas the value of rMSSD is significantly decreased (10 events of apnea), and the value of nHF is significantly decreased (8 events of apnea).

Table 3 Result of HRV during normal respiratory condition and apnea

\begin{tabular}{|c|c|c|c|c|c|c|}
\hline & Mean-RR (ms) & $\mathrm{rMSSD}(\mathrm{ms})$ & $\operatorname{nLF}(\%)$ & $\mathrm{nHF}(\%)$ & $\mathrm{LF} / \mathrm{HF}$ & $\alpha 1$ \\
\hline \multicolumn{7}{|l|}{ Normal } \\
\hline & $928.83 \pm 21.99$ & $35.89 \pm 4.31$ & $38.22 \pm 2.57$ & $61.78 \pm 2.57$ & $0.72 \pm 0.48$ & $0.82 \pm 0.04$ \\
\hline \multicolumn{7}{|c|}{ Respiratory State } \\
\hline $1^{\text {st }}$ Apnea & $966.89 \pm 20.89^{*}$ & $21.54 \pm 2.08^{*}$ & $49.09 \pm 3.8^{*}$ & $50.91 \pm 3.8^{*}$ & $1.55 \pm 0.26^{*}$ & $1.22 \pm 0.09^{*}$ \\
\hline $2^{\text {nd }}$ Apnea & $965.96 \pm 21.85^{*}$ & $20.7 \pm 2.39^{*}$ & $50.85 \pm 3.09^{*}$ & $49.15 \pm 3.09^{*}$ & $1.41 \pm 0.18^{*}$ & $1.21 \pm 0.09 *$ \\
\hline $3^{\text {rd }}$ Apnea & $967.5 \pm 23.21 *$ & $21.19 \pm 2.46^{*}$ & $48.61 \pm 3.39^{*}$ & $51.39 \pm 3.39 *$ & $1.51 \pm 0.29 *$ & $1.16 \pm 0.09 *$ \\
\hline $4^{\text {th }}$ Apnea & $983.39 \pm 22.05^{*}$ & $20.23 \pm 1.76^{*}$ & $48.74 \pm 3.22 *$ & $51.26 \pm 3.22 *$ & $1.25 \pm 0.15^{*}$ & $1.16 \pm 0.09^{*}$ \\
\hline $5^{\text {th }}$ Apnea & $964.61 \pm 20.59^{*}$ & $20.42 \pm 2.32 *$ & $44.87 \pm 4.2$ & $55.13 \pm 4.2$ & $1.48 \pm 0.32 *$ & $1.07 \pm 0.1^{*}$ \\
\hline $6^{\text {th }}$ Apnea & $959.22 \pm 21.76$ & $20.55 \pm 2.16^{*}$ & $51.29 \pm 3.35^{*}$ & $48.71 \pm 3.35^{*}$ & $1.54 \pm 0.22 *$ & $1.17 \pm 0.08^{*}$ \\
\hline $7^{\text {th }}$ Apnea & $964.89 \pm 21.99^{*}$ & $22.36 \pm 2.19 *$ & $46.61 \pm 3.51^{*}$ & $53.38 \pm 3.51 *$ & $1.35 \pm 0.23^{*}$ & $1.3 \pm 0.11^{*}$ \\
\hline
\end{tabular}




\begin{tabular}{|c|c|c|c|c|c|c|}
\hline $8^{\text {th }}$ Apnea & $968.33 \pm 21.46^{*}$ & $22.22 \pm 2.59^{*}$ & $44.75 \pm 4.23$ & $55.25 \pm 4.23$ & $1.42 \pm 0.25^{*}$ & $1.1 \pm 0.09 *$ \\
\hline $9^{\text {th }}$ Apnea & $960.03 \pm 22.11$ & $22.61 \pm 2.38^{*}$ & $50.07 \pm 3.3^{*}$ & $49.93 \pm 3.3^{*}$ & $1.49 \pm 0.26^{*}$ & $1.19 \pm 0.1 *$ \\
\hline $10^{\text {th }}$ Apnea & $961.81 \pm 20.46^{*}$ & $21.79 \pm 2.36^{*}$ & $45.86 \pm 4.03 *$ & $54.13 \pm 4.03 *$ & $1.6 \pm 0.35^{*}$ & $1.19 \pm 0.1 *$ \\
\hline
\end{tabular}

(a)

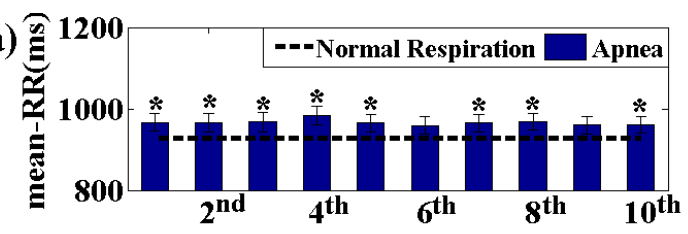

(c)

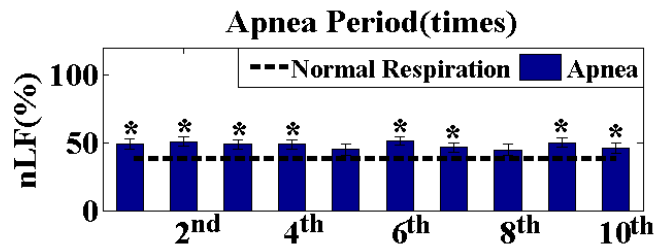

(e)

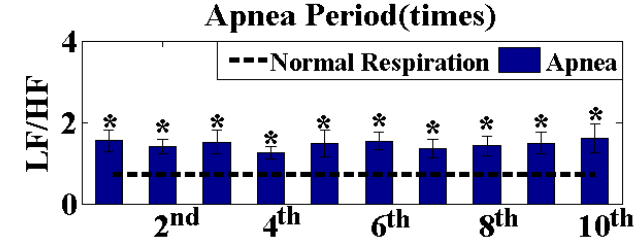

Apnea Period(times) (b)

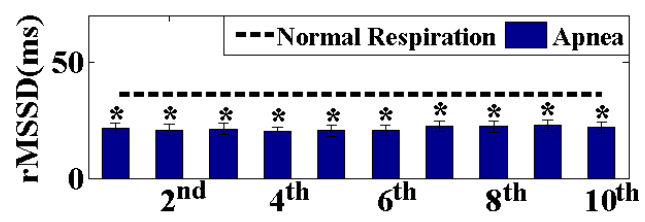

(d)

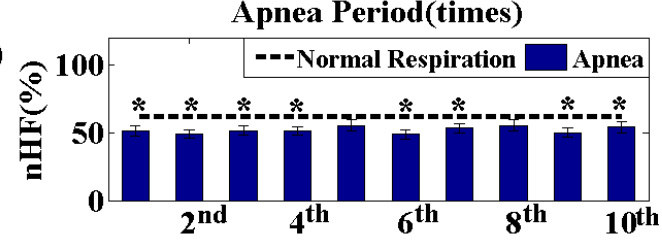

(f)

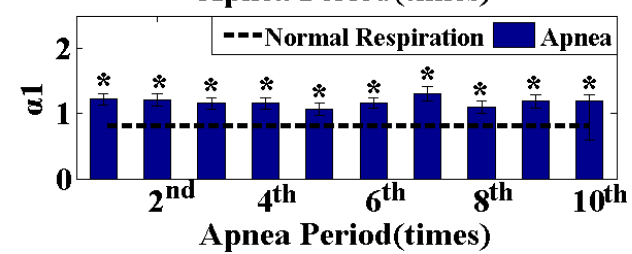

Fig. 4. The values of HRV during normal respiratory condition (dotted line) and apneic state (bar) in study2. (a) the value of

Mean-RR, (b) the value of rMSSD, (c) the value of nLF, (d) the value of nHF, (e) the value of LF/HF, (f) the value of $\alpha 1$. Values

plotted are means \pm SE. Statistical significance between the apneas and normal breathing: $*(\mathrm{P}<0.05)$ significant.

Considering the physiological significance of these HRV indices, we concluded that compared with normal respiration, any form of apnea (simulated or actual apnea) could result in a significant increase in the body's sympathetic activity, a significant decrease in vagal activity, and a significant change in balance of ANS, and it also led to an increase in fractal characteristics of the heart.

\section{Discussion}

In this study, the method of short-term HRV was used to measure and evaluate 
the function of ANS in normal respiration and apneic states of two groups of subjects.

The results showed that compared with the normal respiratory state, simulated apnea (voluntary apnea) and actual apnea (sleep disorder) both lead to a significant increase in the values of the mean-RR, nLF, LF/HF, and $\alpha 1$ whereas the values of rMSSD and nHF both significantly decreased, indicating that sympathetic activity was significantly enhanced, vagal activity was significantly weakened, the balance of ANS produced significant changes, and the fractal characteristics of the heart was enhanced.

There are many methods for evaluating the function of the ANS, such as PTT, Catecholamine assay, Blood pressure monitoring, Radionuclide imaging, and HRV, etc. Among them, the method of PTT needs to find the R-wave of the ECG signal and P-wave of pulse signal to obtain Pulse transit time variability (PPTV), which is complicated to implement. The technique of Catecholamine assay is capable of qualitatively but not quantitatively measuring the function of ANS. The method of blood pressure monitoring and Radionuclide imaging can only accurately measure sympathetic nerve activity, but cannot accurately measure vagal activity. The calculation of HRV is based on the RR interval of the ECG signal. The method of HRV can quantitatively evaluate the function of ANS. It can not only specifically analyze the changes of sympathetic and parasympathetic nerves, but also has the virtues of being non-invasive, simple to calculate, and repeatable [16]. Hence, the method of HRV was chosen to evaluate the function of ANS in our experiment. HRV analysis can be performed using different types of indices. Time domain 
indices can be calculated simply and are intuitive. They have been firstly used in clinical practice, such as mean-RR, rMSSD. The frequency domain indices can measure sympathetho-vagal activity, for example, nHF can reflect vagal activity and nLF can reflect sympathetic activity. Nonlinear domain indices have great advantages in the analysis of nonstationary signal, which can reflect the characteristics of ECG signal changing over time and the fractal characteristics of the heart, such as $\alpha 1$. In our study, time, frequency, and nonlinear domain indices of HRV were used to evaluate the function of ANS from different angles.

The method of Lomb-Scargle was used when we calculated the frequency domain indices of HRV because the experiments involved the analysis of the short-term ECG signal. This method is based on a least squares fit of sinusoids with the calculation performed directly on uneven RR intervals. It does not need to interpolate and resample the original signal, so avoids spectral distortion [17]. Similarly, the method of DFA was chosen when we calculated the nonlinear domain indices because of the short-term ECG signal in our study. The fractal scaling exponent, $\alpha 1$, calculated by DFA can accurately analyze ECG, which is a non-stationary signal, and it can better detect the subtle changes of short-term R-R intervals [18].

The reasons for why apnea leads to arrhythmia are multifaceted and complex. From an anatomical point of view, it has been found that the inspiratory muscles are relaxed in the lungs when apnea occurs. Then the relaxed muscles cause an increase in intra-thoracic pressure and hinders the venous return to the right atrium, which 
reduces the absolute venous pressure. These low-level changes cause the increase of sympathetic activity through low-pressure baroreceptors, which makes ANS unbalanced and eventually leads to arrhythmia [19]. This study explored the causes of arrhythmia from the changes of ANS function. The experimental results showed that when apnea occurs, the human sympathetic activity was enhanced (the value of nLF significantly increased); vagal tone and activity was reduced (the values of rMSSD and nHF significantly decreased); and the original ANS equilibrium state was broken; at the same time, the index of LF/HF reflecting balance of ANS significantly increased too, which further illustrated the imbalance of ANS function. Cardiac activity is regulated by ANS. When the normal function of the ANS is unbalanced, an abnormal heart rhythm is formed, which induces arrhythmia. In addition, some studies have confirmed that the reduction of fractal characteristics of the heart is closely related to the occurrence of cardiac malignant events, such as congestive heart failure, etc. [20], whereas the enhancement of fractal characteristics of the heart would cause arrhythmia [21]. Therefore, when apnea occurred, the phenomenon that the value of $\alpha 1$ reflecting the strength of the fractal characteristics of the heart was significantly enhanced further explained the process of arrhythmia induced by apnea in this study. This study had some limitations. First, although the method of short-term HRV can reflect the changes of ANS during apnea to a certain extent, its accuracy needs to be verified using a more direct method, such as a neural pathways-related approach. Second, most of the simulated and actual apnea in our study lasted for only 20 seconds. Our study only revealed how these 20-second apneic periods influence ANS. 
The relationship between longer apnea and ANS should be investigated further.

\section{Conclusions}

In this study, we compared short-term HRV indices of subjects in normal respiratory and apneic states to analyze whether apnea can affect the balance and normal function of ANS. The results suggest that both form of apnea (simulated or actual apnea) result in a significant increase of sympathetic activity and a decrease of vagal activity, thus leading to an imbalance in function of ANS, as well as an increase in fractal characteristics of the heart. These changes in physiological state are likely to induce and cause the occurrence of arrhythmia. At the same time, we have described algorithms and indices for short-term HRV, which provides a reliable method for future short-term HRV analysis.

\section{Materials and methods}

A. Subjects

Forty-five healthy male $(n=23)$ and female volunteers $(n=22)$, aged 18-35 years and body mass index (BMI) $<21.4 \mathrm{~kg} / \mathrm{m}^{2}$ were enrolled in the study after approval for the study protocol was obtained from the Human Institutional Review Board of the China Medical University. All subjects gave informed consent before the measurements. The study was registered at Chinese Clinical Trial Registry (www.chictr.org.cn) under number ChiCTR-DDD-17014238.

Meanwhile, three hundred subjects were chosen randomly from the dataset of a sleep laboratory for the diagnosis of sleep disorders which is available from PhysioNet (https://physionet.org/physiobank/database/challenge/2018/\#files). The 
subjects had a variety of physiological signals recorded as they slept through the night including: electroencephalography (EEG), electrooculography (EOG), electromyography (EMG), electrocardiology (ECG), oxygen saturation ( $\mathrm{SaO} 2)$, and nasal flow pressure, etc. Subjects were eligible if they maintained continuous steady breathing for more than 5 minutes and experienced apneic periods (> 15 seconds each) more than 10 times during the studied period. Thirty-six male $(\mathrm{n}=11)$ and female $(n=25)$ subjects, aged 40-82 years met the elegibility criteria of our study.

\section{B. Experimental Protocol}

We performed two studies to analyze how apnea influences the ANS using HRV indices: Study1 (45 subjects), in which voluntary apnea was used to simulate apnea and Study2 (36 subjects), in which actual apnea was observed. For Study1 and Study2, absence of nasal flow pressure signal for more than 15 seconds was considered an apneic event [6].

\section{Study 1}

The subjects in the supine position were asked to breathe normally under resting state for 2 minutes. Then the subjects were asked to hold their breathing for about 20 seconds to simulate an apneic event. This type of apnea simulation was repeated every 40 seconds for 10 iterations. During the experiment, we used polysomnography (Alice PDx, Amsterdam, Holland) to collect the physiological signals, including ECG and nasal flow pressure. All signals were acquired synchronously via multiple channels. The sampling rate of the ECG and nasal flow pressure signal is $200 \mathrm{~Hz}$.

Nasal flow pressure was used as the reference signal for dividing normal or 
apneic respiratory events. [Fig.1].

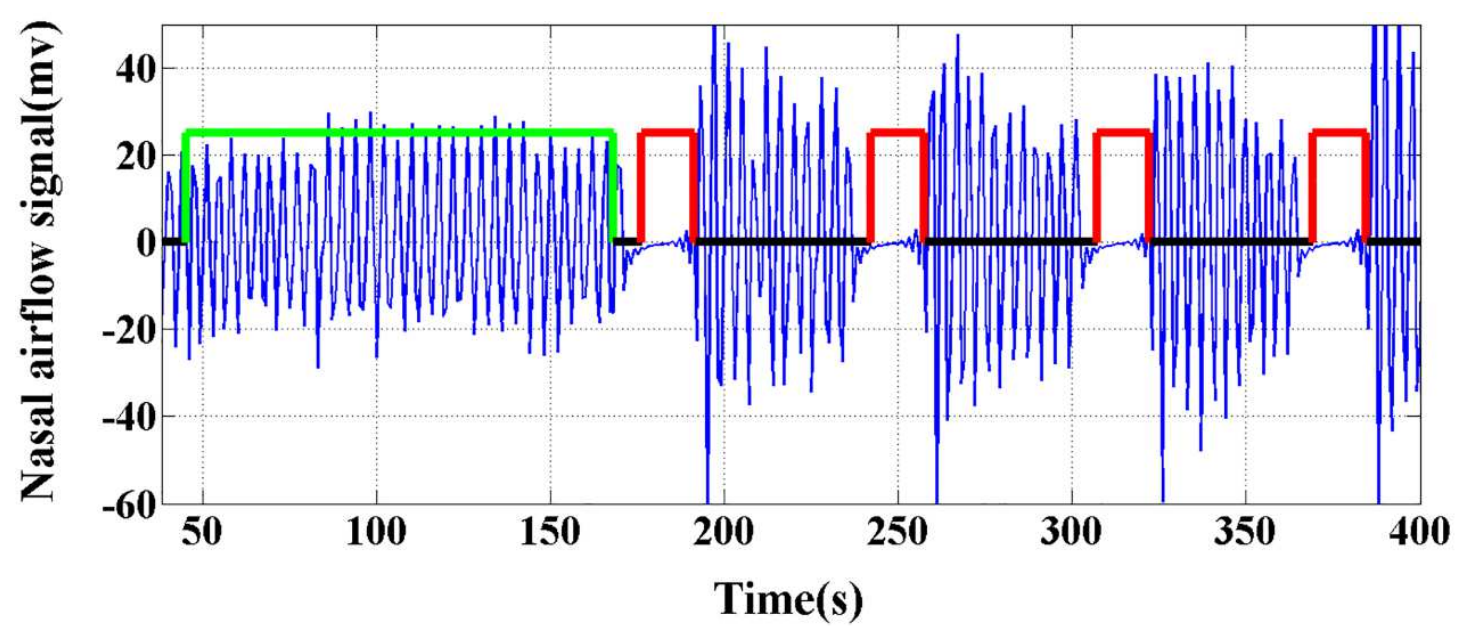

Fig. 1. An example of subject's nasal flow pressure signal in Study1. The green rectangle represents normal respiratory

condition, the red rectangle represents holding simulated apneic event.

HRV analysis was used to evaluate the function of the ANS. In our study, we

used R-R intervals of ECG to calculate the HRV indices. We performed wavelet

transform [7] on the ECG signal (as shown in fig. 2), which removed the baseline wander, ambient noise, and power line interference in the signal, and highlighted the feature of the R-wave. Finally, we identified the R-wave using an amplitude threshold. All the R-waves were validated and checked manually by visual inspection, despite the robustness of the R-wave detection algorithm in a fully automated mode. 
(a)

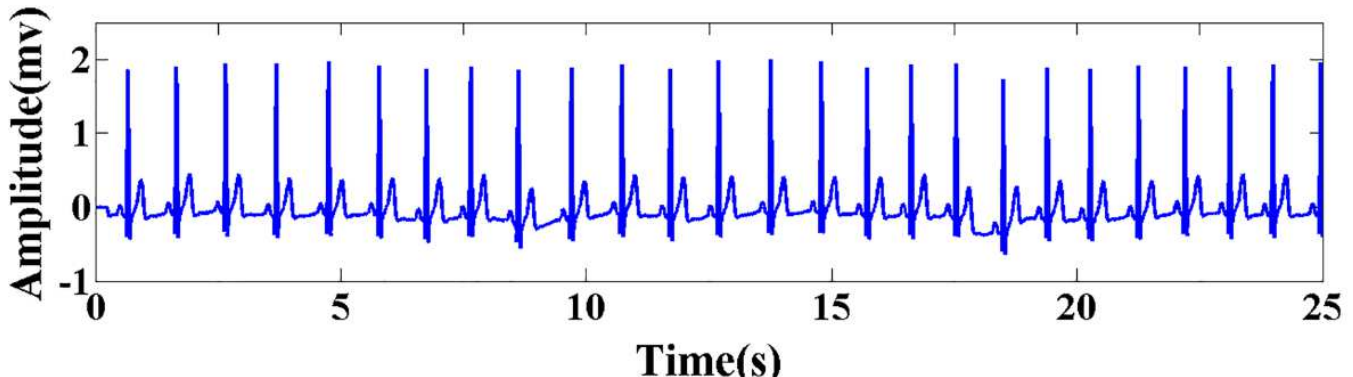

(b)

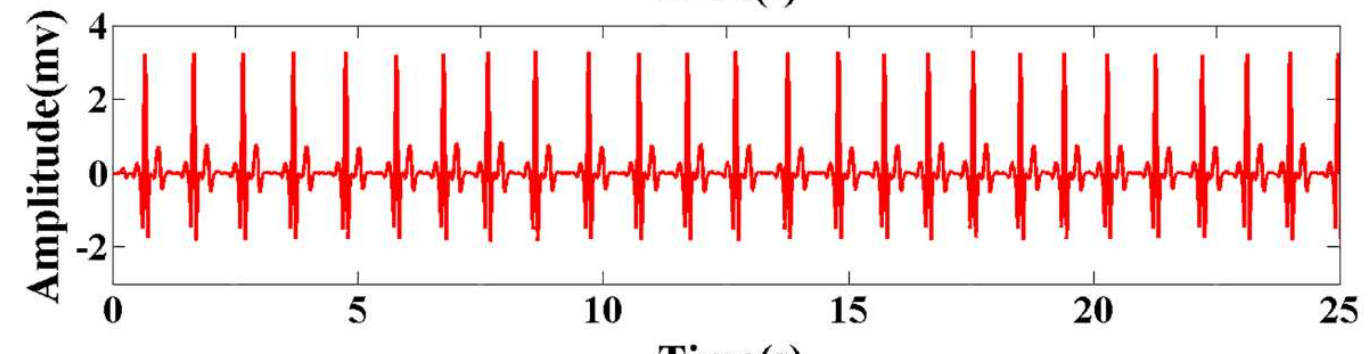

Fig. 2. (a) Original ECG signal (blue), (b) ECG signal after wavelet transform (red).

In order to explore the changes of the ANS during apnea, HRV indices during apneic state (20 seconds) were compared with HRV indices during normal respiratory state (2 minutes). However, some recent research revealed that there were significant differences in certain HRV indices for different lengths ECG signals[8-10].

In order to effectively and reasonably compare the changes in HRV indices under the normal respiration and apneic states, the ECG signals collected from our pilot experiment were used to screen out the HRV indices which did not change with data length changes. These data were collected while the 44 subjects in study 1 were resting with the supine position for a total of 10 minutes. We separately cut the data into 5 minutes, 2 minutes, 40 seconds, and 15 seconds from the initial point of the 10-minute ECG signal, and calculated the HRV indices. The four groups of indices were compared with the HRV indices corresponding to the 10-minute data. If there were no significant differences, the index was considered to be available for subsequent analysis. 

include:

Mean-RR (mean of successive normal R-R intervals): reflects the mean of all R-R intervals, in unit of ms. It can be calculated as:

$$
\operatorname{MEAN}=\overline{\mathrm{RR}}=\sum_{\mathrm{i}=1}^{\mathrm{N}}\left(\mathrm{RR}_{\mathrm{i}} / \mathrm{N}\right)
$$

SDNN (standard deviation of successive normal R-R intervals): reflects the magnitude of sympathetic and parasympathetic tone, in unit of ms. It can be calculated as:

$$
\mathrm{SDNN}=\sqrt{\frac{1}{\mathrm{~N}} \sum_{\mathrm{i}=1}^{\mathrm{N}}\left(\mathrm{RR}_{\mathrm{i}}-\overline{\mathrm{RR}}\right)^{2}}
$$

pNN50 (percent of difference between adjacent successive normal R-R intervals that are greater than $50 \mathrm{~ms}$ ): represents the changes of R-R intervals, reflects vagal activity, in unit of $\%$. It can be calculated as:

$$
\mathrm{pNN} 50=(\mathrm{NN} 50 / \text { TotalNN }) \times 100 \%
$$

rMSSD (square root of the mean squared differences of successive normal R-R intervals): reflects the vagal activity, in unit of ms. It can be calculated as:

$$
\mathrm{rMSSD}=\sqrt{\frac{1}{\mathrm{~N}-1} \sum_{\mathrm{i}=1}^{\mathrm{N}-1}\left(\mathrm{RR}_{\mathrm{i}+1}-\mathrm{RR}_{\mathrm{i}}\right)^{2}}
$$

The frequency domain indices calculated with the method of Lomb-Scargle [12] include:

The value of nHF (normalized units of the power in the low frequency band ranging from 0.04 to $0.15 \mathrm{~Hz}$ ) can reflect vagal activity. nLF (normalized units of the power in the high frequency band ranging from 0.15 to $0.4 \mathrm{~Hz}$ ) is an indicator of sympathetic activity, and its value can relate to the combination of sympathetic and 
parasympathetic function. LF/HF (the ratio of power of LF and HF) is a sensitive indicator of the shift of sympatho-vagal balance [13]. In our study, the windowed Lomb-Scargle method, which had the window width set at 15 seconds with an overlap of 5 seconds, was used to calculate the power spectral distribution.

Nonlinear domain indices include SD1, SD2, and $\alpha 1$. Among them, SD1 and SD2 are obtained based on the method of Poincare plot. SD1 (SD of ellipse width) can reflect the changes of adjacent RR intervals. SD2 (SD of ellipse length) is an indicator to measure the length of Poincare plot, which can reflect the changes of all RR intervals [14]. The fractal scaling exponent $\alpha 1$ calculated by detrended fluctuation analysis (DFA) can not only reflect the sympatho-vagal activity, but also the strength the values of indices decrease significantly with the decrease of data length $(\mathrm{P}<0.05)$, such as SDNN, PNN50, SD1, and SD2, but different data lengths have no significant effects on the values of mean-RR, rMSSD, nLF, nHF, LF/HF, and $\alpha 1(\mathrm{P}>0.05)$. Table 1 Comparison of HRV indices with different data lengths under the normal of the fractal characteristics of the heart. It is often used as an indicator to predict the occurrence of heart disease [15].

The screening results of HRV indices are shown in Table 1 . We observed that

\begin{tabular}{lccccc}
\hline HRV indices & $10 \mathrm{~min}$ & $5 \mathrm{~min}$ & $2 \mathrm{~min}$ & $40 \mathrm{~s}$ & $15 \mathrm{~s}$ \\
\hline Mean-RR (ms) & $870.49 \pm 19.2$ & $867.16 \pm 18.83$ & $864.9 \pm 18.89$ & $870.61 \pm 21.44$ & $874.51 \pm 22$ \\
& & & & & \\
SDNN (ms) & $53.34 \pm 3.9$ & $50.35 \pm 4.1^{*}$ & $49.26 \pm 4.66$ & $43.82 \pm 5.17^{*}$ & $41.86 \pm 5.44 *$ \\
pNN50 (\%) & $20.85 \pm 2.9$ & $21.22 \pm 3$ & $21.05 \pm 3.16$ & $17.37 \pm 2.95^{*}$ & $15.8 \pm 3.12^{*}$ \\
\hline
\end{tabular}




\begin{tabular}{|c|c|c|c|c|c|}
\hline rMSSD (ms) & $43.41 \pm 3.79$ & $41.59 \pm 3.95$ & $41.1 \pm 4$ & $40.63 \pm 5.17$ & $39.28 \pm 5.22$ \\
\hline $\mathrm{nLF}(\%)$ & $47.88 \pm 2.66$ & $47.95 \pm 2.68$ & $50.68 \pm 2.92$ & $45.83 \pm 3.29$ & $43.79 \pm 3.11$ \\
\hline $\mathrm{nHF}(\%)$ & $52.12 \pm 2.66$ & $52.05 \pm 2.68$ & $49.34 \pm 2.92$ & $54.17 \pm 3.29$ & $56.21 \pm 3.11$ \\
\hline $\mathrm{LF} / \mathrm{HF}$ & $1.24 \pm 0.17$ & $1.32 \pm 10.17$ & $1.46 \pm 0.19$ & $1.37 \pm 0.23$ & $1.13 \pm 0.16$ \\
\hline SD1 & $37.7 \pm 2.75$ & $35.57 \pm 2.89 *$ & $34.77 \pm 3.26$ & $31.06 \pm 3.7 *$ & $26.95 \pm 3.57 *$ \\
\hline $\mathrm{SD} 2$ & $65.34 \pm 4.79$ & $61.69 \pm 5.03 *$ & $60.37 \pm 5.72$ & $53.62 \pm 6.3^{*}$ & $47.39 \pm 6.04^{*}$ \\
\hline$\alpha 1$ & $0.96 \pm 0.03$ & $0.95 \pm 0.03$ & $0.97 \pm 0.04$ & $0.93 \pm 0.05$ & $1.07 \pm 0.07$ \\
\hline
\end{tabular}

Values are expressed as the mean \pm SE. Statistical significance between other length of respiratory condition with

length of 10 minutes: * $(\mathrm{P}<0.05)$ significant.

Therefore, we calculated and compared the six HRV indices of 45 subjects in the states of normal respiration ( 2 minutes) and voluntary apnea (20 seconds) to evaluate the effects on ANS during voluntary apnea, respectively. These six HRV indices were also used in study2.

Study 2

For the enrolled thirty-six subjects, we selected the 5-minute periods when the subjects maintained the normal breathing state and the periods when apnea appeared 10 times during the sampling period. The ECG and nasal flow pressure signals during these periods were used for subsequent analysis. Nasal flow pressure signal was used as the reference signal to divide the samples into different respiratory states: normal 279 or apneic.

HRV analysis is the method we adopt to evaluate the function of ANS. In study2, we also used R-R intervals of ECG to calculate HRV indices. The R-R intervals were 
determined in the same way as in study1.

Just like in study1, we calculated and compared the six HRV indices of the 36 subjects in normal respiratory (5 minutes) and apneic states (>15 seconds) to evaluate its influence on the balance and normal function of ANS during apnea, respectively. In study 1 and study2, the analysis and processing of the signals were performed using MATLAB (version 8.3, Natick, Massachusetts, USA) software.

C. Statistical analysis

When HRV indices were screened in study1, we used the method of Paired-sample t-tests to compare the HRV indices for 5 minutes, 2 minutes, 40 seconds, and 15 seconds to the HRV indices for 10 minutes. We used the method of Paired-sample t-tests to compare the six HRV indices under normal respiratory state (5 minutes) to the indices in apneic state ( $>15$ seconds). All data were calculated by SPSS (version 22.0, Inc. Chicago, IL, USA) software, and they were presented in the form of mean $\pm \mathrm{SE}$. At the same time, $\mathrm{P}=0.05$ was defined. When $\mathrm{P}<0.05$, the two groups of data had statistically significant difference. When $\mathrm{P}>0.05$, there was no statistically significant difference between the two groups.

\section{Abbreviations}

ANS, autonomic nervous system; HRV, heart rate variability; ECG, electrocardiology; Mean-RR ,mean of successive normal R-R intervals; SDNN, standard deviation of successive normal R-R intervals; pNN50, percent of difference between adjacent successive normal R-R intervals that are greater than $50 \mathrm{~ms}$; rMSSD, square root of the mean squared differences of successive normal R-R intervals; nHF, normalized 
units of the power in the low frequency band ranging from 0.04 to $0.15 \mathrm{~Hz}$; nLF, normalized units of the power in the high frequency band ranging from 0.15 to $0.4 \mathrm{~Hz}$; LF/HF, the ratio of power of LF and HF; SD1, SD of ellipse width; SD2, SD of ellipse length; DFA, detrended fluctuation analysis.

Ethics approval and consent to participate

All subjects gave informed consent before the measurements in study1. The study was

ChiCTR-DDD-17014238.

\section{Consent for publication}

313 All subjects have consent for publication.

\section{Availability of data and materials}

\section{Funding}

This work was supported by grant No. 81401485 from the National Natural Science Foundation of China, Beijing, P.R. China.

\section{Authors' contributions}

LY contributed conception and design of the study, data analyzation, and article 
drafting; $\mathrm{BH}$ and WL contributed equally to the literature search, data analyzation, and article drafting; XZ performed the statistical analysis and data analyzation; YW and $\mathrm{BH}$ participated in data collection and information organization; LB contributed to and article drafting and study design. All authors read and approved the final manuscript.

\section{Acknowledgements}

The research described in this paper was supported by the National Natural Science Foundation of China (Grant No. 81401485).

\section{References}

[1] Ar1 H, Arı S, Yazıcı F, Koca V, Bozat T. Cardiac autonomic function and cardiac arrhythmias in patients with obstructive sleep apnea. Turk Kardiyol Dern Ars. 2011. 39(4): 292-9.

[2] Lindholm P, Lundgren CE. The physiology and pathophysiology of human breath-hold diving. J Appl Physiol (1985). 2009. 106(1): 284-92.

[3] Hersi AS. Obstructive sleep apnea and cardiac arrhythmias. Ann Thorac Med. 2010. 5(1): 10-17.

[4] Shen MJ, Zipes DP. Role of the autonomic nervous system in modulating cardiac arrhythmias. Circ Res. 2014. 114(6): 1004-21.

[5] Smith RP, Veale D, Pépin JL, Lévy PA. Obstructive sleep apnoea and the autonomic nervous system. Sleep Med Rev. 1998. 2(2): 69-92.

[6] Liu J, Ai C, Zhang B, et al. Tracheal sounds accurately detect apnea in patients recovering from anesthesia. J Clin Monit Comput. 2018 . 
[7] Shinar Z, Baharav A, Dagan Y, Akselrod S. Automatic detection of slow-wave-sleep using heart rate variability. 2001 .

[8] Smith AL, Owen H, Reynolds KJ. Heart rate variability indices for very short-term (30 beat) analysis. Part 1: survey and toolbox. J Clin Monit Comput. 2013. 27(5): 569-76.

[9] Smith AL, Owen H, Reynolds KJ. Heart rate variability indices for very short-term (30 beat) analysis. Part 2: validation. J Clin Monit Comput. 2013. 27(5): 577-85.

[10] Chen X, Chen T, Yun F, Huang Y, Li J. Effect of repetitive end-inspiration breath holding on very short-term heart rate variability in healthy humans. Physiol Meas. 2014. 35(12): 2429-2445.

[11] Heart rate variability: Origins, methods, and interpretive caveats[J]. Psychophysiology. 1997. 34(6):623-648.

[12] Laguna P, Moody GB, Mark RG. Power spectral density of unevenly sampled data by least-square analysis: performance and application to heart rate signals. IEEE Trans Biomed Eng. 1998. 45(6): 698-715.

[13] Malik M, Bigger JT, Camm AJ, et al. Heart rate variability. Standards of measurement, physiological interpretation, and clinical use. Task Force of the European Society of Cardiology and the North American Society of Pacing and Electrophysiology. Circulation. 1996. 93(5): 1043-65.

[14] Brennan M, Palaniswami M, Kamen P. Do existing measures of Poincare plot geometry reflect nonlinear features of heart rate variability. IEEE Trans 
Biomed Eng. 2001. 48(11): 1342-7.

371 [15] Tulppo MP, Kiviniemi AM, Hautala AJ, et al. Physiological background of the loss of fractal heart rate dynamics. Circulation. 2005. 112(3): 314-9.

[16] Sztajzel J. Heart rate variability: a noninvasive electrocardiographic method to measure the autonomic nervous system. Swiss Med Wkly. 2004. 134(35-36): 514-22.

[17] Smith AL, Owen H, Reynolds KJ. Can short-term heart rate variability be used to monitor fentanyl-midazolam induced changes in ANS preceding respiratory depression. Journal of Clinical Monitoring \& Computing. 2014. 29(3): 1-13.

[18] Perkiömäki JS, Zareba W, Kalaria VG, Couderc JP, Huikuri HV, Moss AJ. Comparability of nonlinear measures of heart rate variability between longand short-term electrocardiographic recordings is. Am J Cardiol. 2001. 87(7): 905-908.

[19] Macefield VG, Gandevia SC, Henderson LA. Neural sites involved in the sustained increase in muscle sympathetic nerve activity induced by inspiratory capacity apnea: a fMRI study. J Appl Physiol (1985). 2006. 100(1): 266-273.

[20] Peng C kg, Havlin S, Stanley HE, Goldberger AL. Quantification of scaling exponents and crossover phenomena in nonstationary heartbeat time series. Chaos. 1995. 5(1): 82-87.

[21] Rodriguez E, Lerma C, Echeverria JC, Alvarez-Ramirez J. ECG scaling properties of cardiac arrhythmias using detrended fluctuation analysis. Physiol 


\section{Figures}

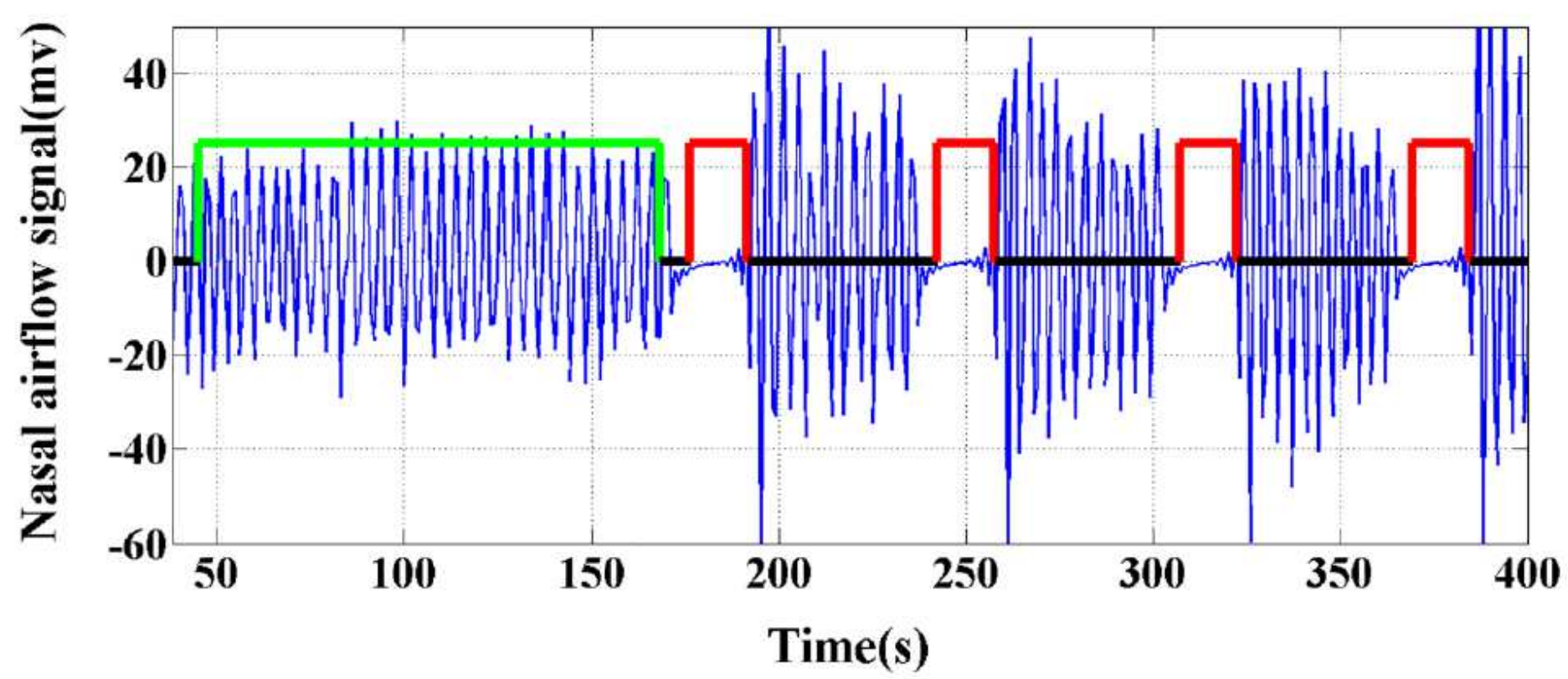

Figure 1

An example of subject's nasal flow pressure signal in Study1. The green rectangle represents normal respiratory condition, the red rectangle represents holding simulated apneic event.

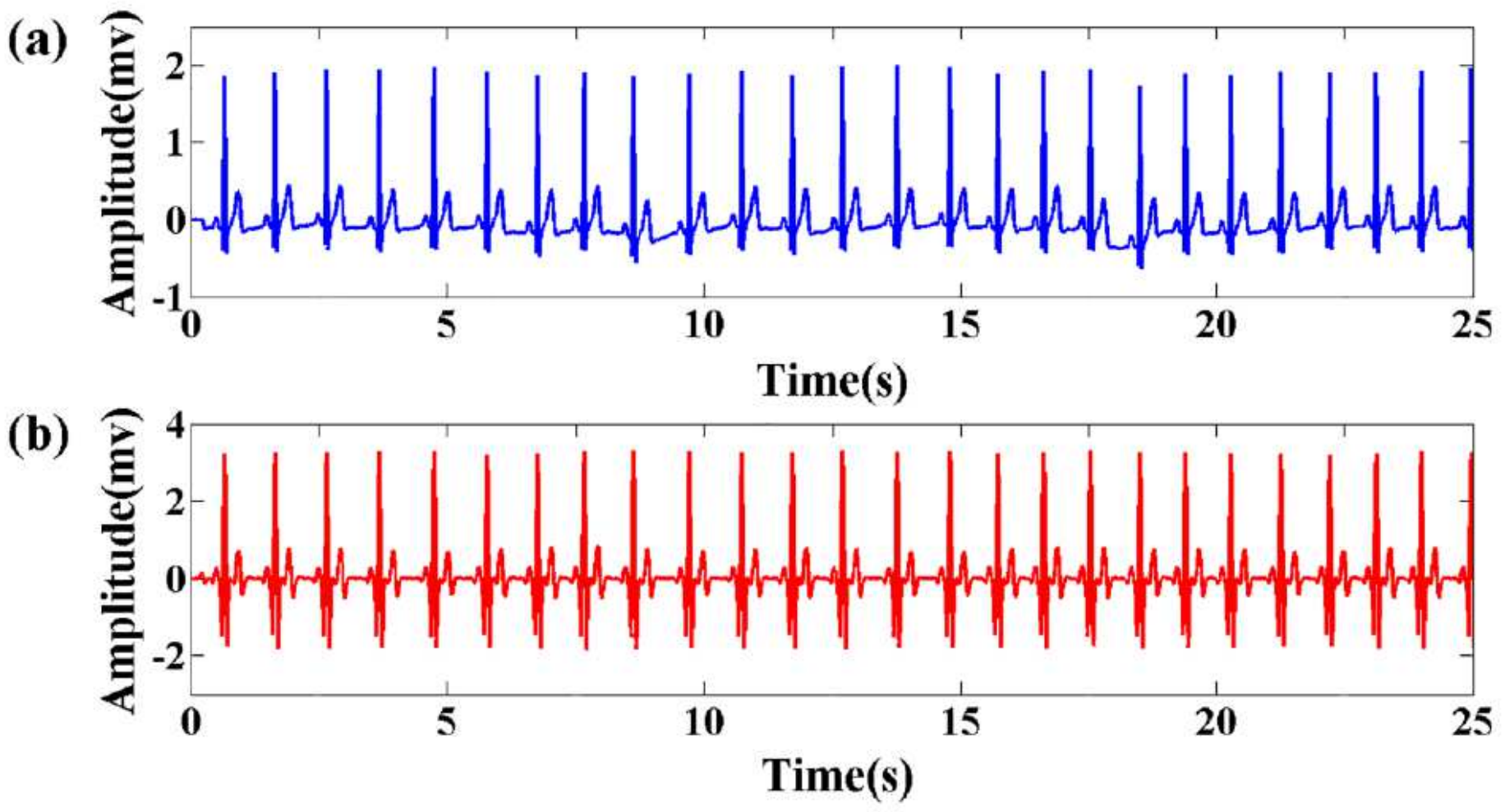

Figure 2 
(a) Original ECG signal (blue), (b) ECG signal after wavelet transform (red).

(a)

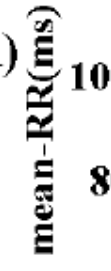

800

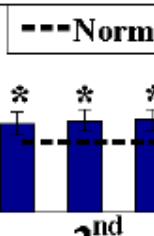

$2^{\text {nd }}$

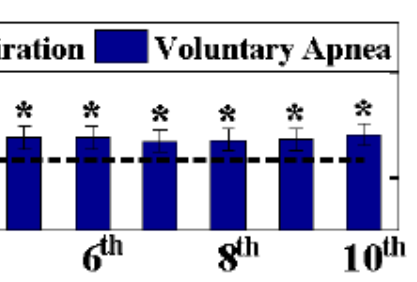

Voluntary Apneic Period(times)

(c)

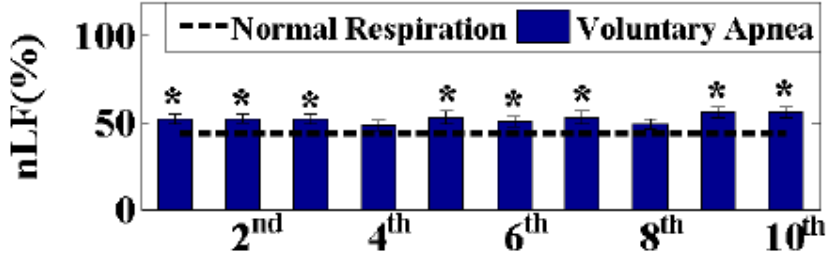

(e)

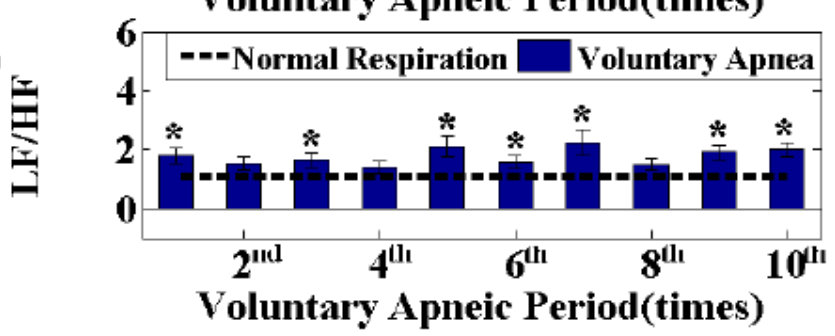

(b)

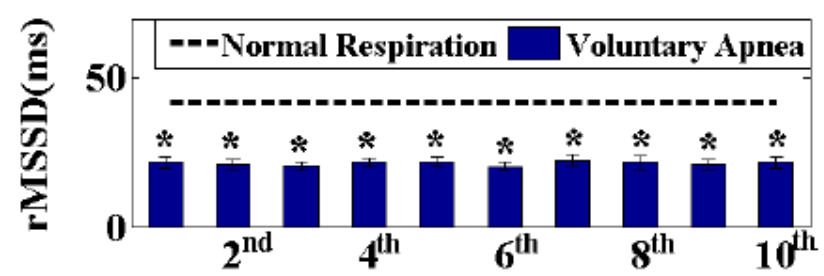

Voluntary Apneic Period(times)

(d)

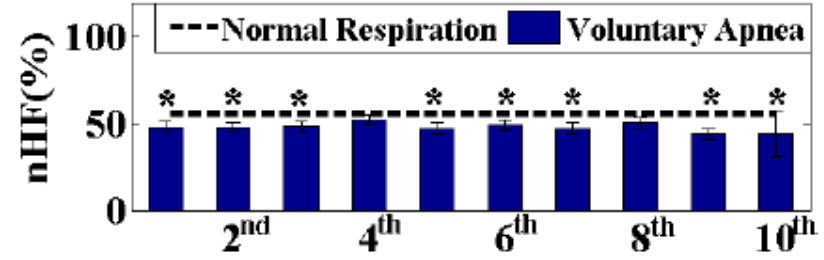

(f)

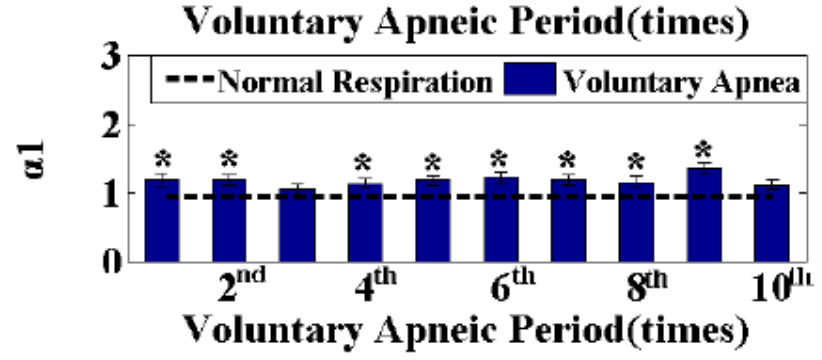

Figure 3

The values of HRV during normal respiratory condition (dotted line) and voluntary apneic state (bar) in study1. (a) the value of Mean-RR, (b) the value of rMSSD, (c) the value of $n L F,(d)$ the value of $n H F,(e)$ the value of $L F / H F,(f)$ the value of a1. Values plotted are means \pm SE. Statistical significance between the voluntary apneas and normal breathing: * $(P<0.05)$ significant. 
(a)

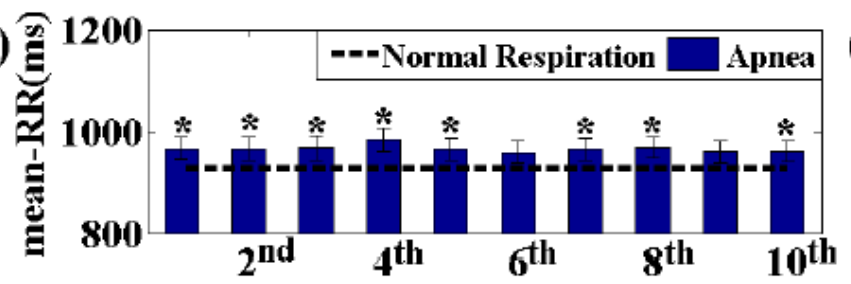

(c)

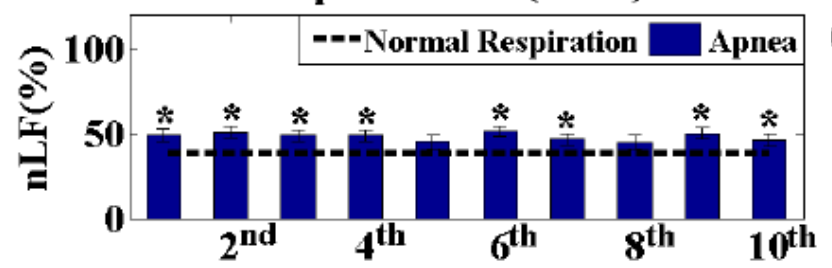

(e)

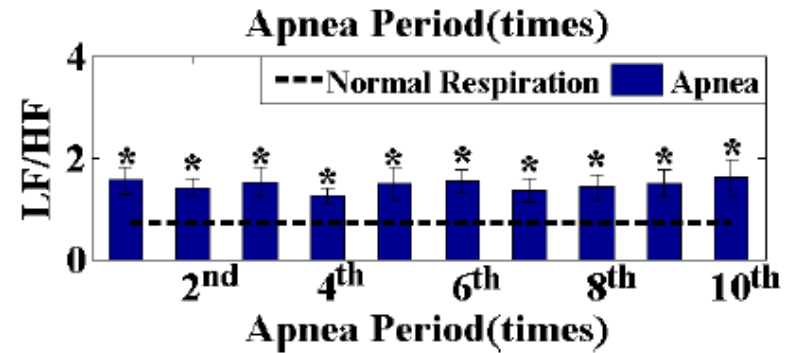

(b)

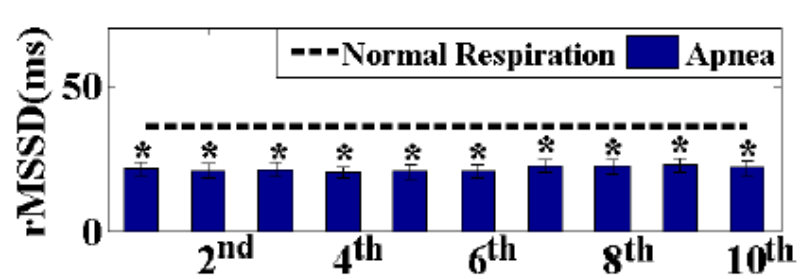

(d)

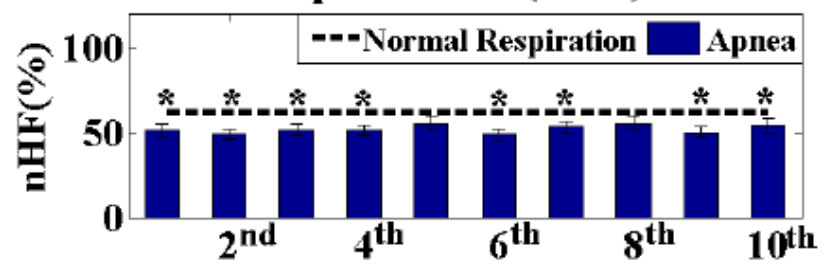

(f)

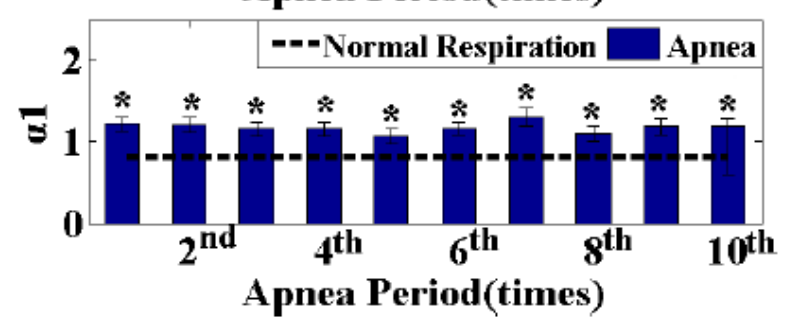

Figure 4

The values of HRV during normal respiratory condition (dotted line) and apneic state (bar) in study2. (a) the value of Mean-RR, (b) the value of rMSSD, (c) the value of $n L F,(d)$ the value of $n H F,(e)$ the value of $\mathrm{LF} / \mathrm{HF}$, (f) the value of a1. Values plotted are means $\pm \mathrm{SE}$. Statistical significance between the apneas and normal breathing: * $(P<0.05)$ significant. 